\title{
A case report of intracranial hypertension and aseptic meningitis: anti-tumor necrosis factor associated or juvenile idiopathic arthritis related
}

\author{
Ümmüşen Kaya Akça ${ }^{1 \oplus}$, Okan Sökmen ${ }^{2 \oplus}$, Ertuğrul Çağrı Bölek ${ }^{3 \oplus}$, Selcan Demir ${ }^{1 \oplus,}$ \\ Levent K1lıç ${ }^{3 \oplus}$, Işın Ünal Çevik ${ }^{2 \oplus}$, Yelda Bilginer ${ }^{1 \oplus}$ \\ ${ }^{1}$ Division of Pediatric Rheumatology, Department of Pediatrics, Departments of ${ }^{2}$ Neurology and ${ }^{3}$ Rheumatology, Hacettepe University \\ Faculty of Medicine, Ankara, Turkey.
}

\begin{abstract}
Background. The adverse effects of tumor necrosis factor alpha inhibitors (TNFi) are well characterized but rare adverse events are increasing day by day.

Case. We presented an 18-year-old girl with rheumatoid factor positive polyarticular juvenile idiopathic arthritis (JIA) who developed fever, headache, and nausea after the second dose of adalimumab. In addition to her suspicious complaints for meningitis, she had bilateral papilledema and partial abducens nerve palsy. Leptomeningeal contrast enhancement was noted in magnetic resonance imaging (MRI) of the brain. Brain MRI venography was normal. The cerebrospinal fluid (CSF) opening pressure was high but CSF analysis was normal. She was diagnosed with non-infectious subacute meningitis. Since brain biopsy was not performed, no definite distinction could be made between TNFi related aseptic meningitis or cerebral involvement of JIA. Due to the onset of neurological complaints after initiation of adalimumab treatment and rare cerebral involvement in JIA, the drug-associated aseptic meningitis was likely to be responsible in our patient. Adalimumab was discontinued and methylprednisolone followed by methotrexate treatment were initiated. Her symptoms resolved and control brain MRI was normal.
\end{abstract}

Conclusion. Pediatric rheumatologists should be aware of this potentially severe side effect of anti-TNF treatment.

Key words: aseptic meningitis, intracranial hypertension, tumor necrosis factor alpha inhibitors.

Tumor necrosis factor alpha inhibitors (TNFi) are used in many inflammatory diseases including juvenile idiopathic arthritis (JIA), rheumatoid arthritis, inflammatory bowel diseases, and psoriasis. Despite their clinical effectiveness, various adverse events have been reported with their increasing use. While demyelination is a well-known neurological adverse effects of TNFi, TNFi-associated aseptic meningitis (TAAM) is a rare and serious complication. ${ }^{1,2}$ Booker et al. ${ }^{3}$ reported a patient who was treated

Yelda Bilginer

yeldabilginer@yahoo.com

Received 13th January 2020, revised 2nd June 2020, accepted 12th September 2020. with etanercept for rheumatoid arthritis and presented with aseptic meningitis. A literature review reported 10 adult patients who used antiTNF with different diagnoses and developed aseptic meningitis. Despite reported cases in adults, to our knowledge, aseptic meningitis has not been reported in children using antiTNF therapy in the literature.

The mechanisms underlying TAAM have not been fully elucidated. Since the anti-TNF agents cannot cross the blood-brain barrier, the drugrelated direct toxic effect is not considered. ${ }^{4}$ It may be due to a delayed hypersensitivity reaction or peripheral inhibition of TNF alpha which may lead to upregulation of proinflammatory pathways in the brain. 
In our study, we presented a patient with rheumatoid factor positive JIA who most likely developed TNFi-related aseptic meningitis.

\section{Case Report}

A 18-year-old girl has been followed up for 4 years with the diagnosis of rheumatoid factor positive polyarticular JIA. In the first 6 months of the disease, she had active arthritis on bilateral elbow, wrists, and ankle joints. She used non-steroidal anti-inflammatory drugs and methotrexate for ten months. Etanercept treatment was started due to disease activity (JADAS: 10) and was used for 1.5 years. In her follow-up, etanercept was switched to adalimumab (40 mg every 2 weeks) due to persistent disease activity (JADAS: 16). After the second dose of adalimumab, she complained of progressive dull, throbbing type headache, aggravated by Valsalva maneuvers, lying down, worse at night, accompanied by photophobia, phonophobia, and blurred vision. Headaches had become constant for the last 10 days and headache severity was 9/10 (VRS: verbal rating scale). She also reported fever which started after second dose of adalimumab, lasted for one day, and reached to $38.7^{\circ} \mathrm{C}$. She had arthralgia and swelling in the right knee and left ankle joint. She did not use any other medication except adalimumab. On physical examination, her blood pressure was $110 / 70$ $\mathrm{mmHg}\left(<50\right.$ th percentile) and fever was $37.8^{\circ} \mathrm{C}$. On neurological examination, there were no signs of meningeal irritation or nuchal rigidity. Bilateral papilledema and partial abducens palsy were noted. Motor, sensory, reflex, cerebellar, and extrapyramidal system examinations were normal. She had active arthritis in the right knee and left ankle joint. Examination of other systems were unremarkable. Laboratory test results revealed normal leukocyte count $\left(7,100 / \mathrm{mm}^{3}\right.$ [4.1-11.2]) and blood biochemistry, increased erythrocyte sedimentation rate (73 $\mathrm{mm} /$ hour [0-25]) and C-reactive protein levels (12.3 $\mathrm{mg} / \mathrm{dl}$ [0-0.8]). In contrast enhanced brain magnetic resonance imaging (MRI), leptomeningeal enhancement was identified (Fig. 1). The cerebral venous sinuses were normal on brain MRI venography. Lumbar puncture was performed. The cerebrospinal fluid (CSF) appearance was clear however the opening pressure was high $(250 \mathrm{~mm})$. The
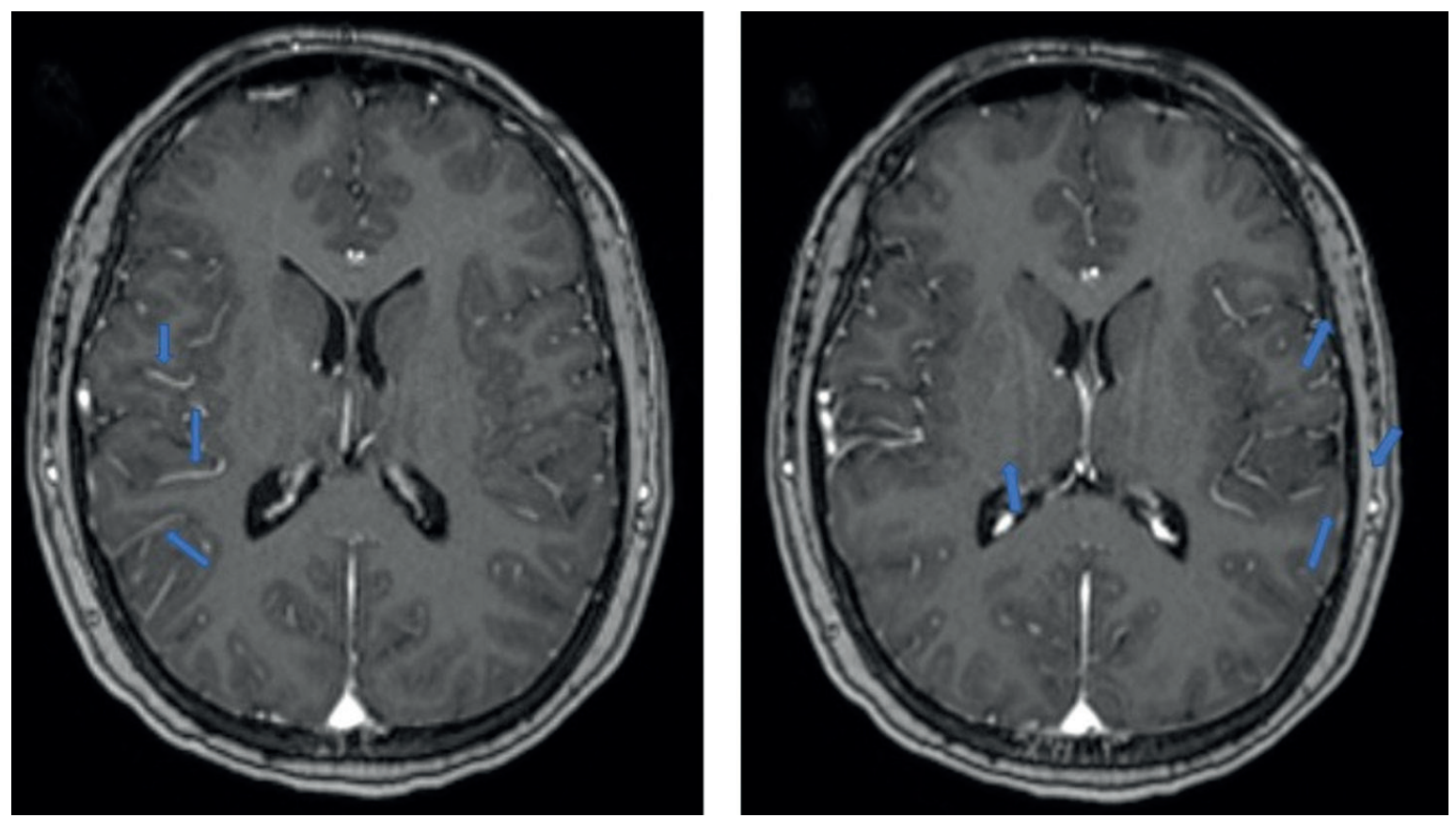

Fig. 1. Leptomeningeal enhancement on contrast enhanced brain magnetic resonance imaging (arrows). 
CSF protein level was normal $(20 \mathrm{mg} / \mathrm{dl})$. The CSF glucose level was $70 \mathrm{mg} / \mathrm{dl}$ and blood glucose level was $91 \mathrm{mg} / \mathrm{dl}$. On microscopic examination, few white blood cells were noted. CSF cultures were negative. CSF antibodies against infectious agents (measles, toxoplasma, rubella, cytomegalovirus, Epstein-Barr virus, mumps virus, herpes simplex virus, borrelia, brucella, varicella zoster virus, cryptococcus, and Treponema pallidum) were all negative. She was diagnosed with non-infectious subacute aseptic meningitis. Adalimumab treatment was discontinued and methylprednisolone (48 $\mathrm{mg} /$ day) treatment was started. Acetazolamide (1000 mg/day) and topiramate (100 mg/day) for intracranial hypertension and methotrexate (15 $\mathrm{mg} /$ week) for polyarticular JIA were initiated in the follow up. Clinical signs of arthritis improved on the 6th day of treatment. At the end of the first month, acute phase reactants were completely normalized. One month after discharge, the patient was free of headache.

Informed consent was received from the patient and her parents for this report.

\section{Discussion}

To our knowledge, this is the first reported case of a child with rheumatologic disease with possibly anti-TNF related aseptic meningitis and intracranial hypertension. Aseptic meningitis has been reported in adult patients receiving TNFi as an uncommon adverse reaction. Case reports of aseptic meningitis associated with adalimumab, etanercept and infliximab treatment in patients with rheumatoid arthritis have been published. ${ }^{3,6-8}$ In addition to rheumatoid arthritis, TAAM has also been reported in patients with psoriatic arthritis, inflammatory bowel disease and psoriasis. ${ }^{9-11}$ Simultaneously, our patient had intracranial hypertension. The clinical characteristics of intracranial hypertension were the presence of headache, pulsatile tinnitus, transient visual obscurations, 6th cranial palsy and papilledema. The three main principles of treatment were to treat the underlying disease, to protect the vision and to minimize the headache. ${ }^{12}$ Acetazolamide and topiramate treatments were initiated to resolve headaches and to reduce intracranial pressure.

Patients taking biologic agents are at higher risk for infections. Meningitis especially related to Listeria monocytogenes infection have been reported in patients treated with anti-TNF drugs. ${ }^{13}$ However, it is not easy to distinguish whether it is drug-related or co-incidental. Aseptic meningitis should be considered in patients with meningeal symptoms when CSF culture results are negative. Our patient was also diagnosed with aseptic meningitis due to meningeal symptoms, leptomeningeal enhancement, and lack of identifiable microorganism in the CSF. Although high leukocyte count, normal glucose, and elevated protein CSF levels are usually observed in patients with aseptic meningitis, MatsuuraOtsuki et al. ${ }^{14}$ reported normal CSF findings of a patient with psoriatic arthritis who developed infliximab-associated aseptic meningitis. ${ }^{15}$ Pashankar et al. ${ }^{16}$ also found normal CSF results in a child that presented with aseptic meningitis after co-trimoxazole treatment. It has been reported that many drugs lead to aseptic meningitis. Nonsteroidal anti-inflammatory drugs, antimicrobials, intravenous immunoglobulin, intrathecal agents and vaccines are the biggest culprit agents. ${ }^{17}$

There have been rare case reports of cerebral involvement in juvenile idiopathic arthritis. Jan et al. ${ }^{18}$ reported in 1972 that 13 of 170 patients with juvenile idiopathic arthritis had signs of CNS dysfunction. They found EEG abnormalities during the acute toxic stages in many cases and other manifestations of toxic encephalopathy such as irritability, drowsiness, stupor, convulsions, and marked meningismus in severe cases. However, they ruled out meningitis with normal CSF findings. In adults, rheumatoid meningitis, a form of aseptic meningitis, is a rare well known extraarticular manifestations of rheumatoid arthritis. Rheumatoid meningitis has been reported in patients with a long-standing history 
of seropositive RA, albeit rare. ${ }^{19}$ It usually develops in long-term follow-up. Rheumatoid meningitis has been proven with brain biopsy in some cases. A necrotizing granulomatous meningitis which is identical for rheumatoid nodules has also been shown. ${ }^{20}$ Our patient had rheumatoid factor positive JIA which has been considered as the early onset of adult RA. Since the onset of neurological complaints occurred after adalimumab treatment, drug induced aseptic meningitis was likely to be responsible for our case. Biopsy which was the diagnostic limitation in our patient was necessary for the precise separation of these two diagnoses.

Discontinuation of the drug responsible for drug-related aseptic meningitis is the basis of treatment. It was reported that systemic steroid treatment can be used in drug induced aseptic meningitis. ${ }^{21}$ The time to resolution might take place until 21 days after discontinuation of the drug. ${ }^{4}$ We also discontinued adalimumab treatment and began methylprednisolone treatment. Clinical recovery occurred within two weeks after drug cessation.

In conclusion, we presented a child with intracranial hypertension and aseptic meningitis possibly induced by adalimumab treatment. It is important for pediatric rheumatologists to be aware of this potentially severe side effect of TNFi treatment. It may be necessary to perform a more detailed examination in patients using biological agents who present with neurological symptoms.

\section{Author contribution}

The authors confirm contribution to the paper as follows: study conception and design: Ümmüşen Kaya Akça, Yelda Bilginer; data collection: Ümmüşen Kaya Akça, Okan Sökmen, Ertuğrul Çağrı Bölek, Selcan Demir; analysis and interpretation of results: Levent Kılıç, Yelda Bilginer, Işıl Ünal Çevik; draft manuscript preparation: Yelda Bilginer. All authors reviewed the results and approved the final version of the manuscript.

\section{Conflict of interest}

The authors declare that there is no conflict of interest regarding the publication of this article.

\section{REFERENCES}

1. van Oosten BW, Barkhof F, Truyen L, et al. Increased MRI activity and immune activation in two multiple sclerosis patients treated with the monoclonal antitumor necrosis factor antibody cA2. Neurology 1996; 47: 1531-1534.

2. Arnason B, Jacobs G, Hanlon M, et al; The Lenercept Multiple Sclerosis Study Group and The University of British Columbia MS/MRI Analysis Group. TNF neutralization in MS-results of a randomized, placebo-controlled multicenter study. Neurology 1999; 53: 457-465.

3. Booker MJ, Flint J, Saravana S. Aseptic meningitis in a patient taking etanercept for rheumatoid arthritis: a case report. Cases J 2008; 1: 364.

4. Wang DY, Chong WS, Pan JY, Heng YK. First case report of aseptic meningitis induced by adalimumab administered for treatment of chronic plaque psoriasis. J Investig Allergol Clin Immunol 2017; 27: 183-185.

5. Junga Z, Theeler B, Singla M. Infliximab-induced aseptic meningitis in a patient with Crohn's disease. ACG Case Rep J 2018; 5: e48.

6. Jazeron A, Lallier JC, Rihn B, Thiercelin MC. Aseptic meningitis possibly induced by adalimumab. Joint Bone Spine 2010; 6: 618-619.

7. López Méndez PL, Martín Santana I, del Pino Reyes Yánez M, , Ruano Hernández A, Hernández Beriain JÁ, Hervás García M. Meningeal and Guillain-Barrè syndrome in a patient with rheumatoid arthritis receiving adalimumab therapy. Reumatol Clin 2011; 7: 401-403.

8. Marotte H, Charrin JE, Miossec P. Infliximabinduced aseptic meningitis. Lancet 2001; 358: 1784.

9. Honda Y, Otsuka A, Egawa G, et al. Multiple neurological abnormalities, including pontine hemorrhage, multiple sclerosis and aseptic meningitis, during anti-TNF- $\alpha$ therapy in psoriatic arthritis. Eur J Dermatol 2015; 25: 487-488.

10. Manthey C, Lohse AW, Pace A. Case report of aseptic meningitis in a patient with Crohn's disease under infliximab therapy. Inflamm Bowel Dis 2011; 17: E10.

11. Shah R, Shah M, Bansal N, Manocha D. Infliximabinduced aseptic meningitis. Am J Emerg Med 2014; 32: 1560.e3-4. 
12. Mollan SP, Davies B, Silver NC, et al. Idiopathic intracranial hypertension: consensus guidelines on management. J Neurol Neurosurg Psychiatry 2018; 89: 1088-1100.

13. Peña-Sagredo JL, Hernández MV, Fernandez-Llanio $\mathrm{N}$, et al; Biobadaser Group. Listeria monocytogenes infection in patients with rheumatic diseases on TNF-alpha antagonist therapy: the Spanish Study Group experience. Clin Exp Rheumatol 2008; 26: 854-859.

14. Matsuura-Otsuki Y, Hanafusa T, Yokozeki H, Watanabe K. Infliximab-induced aseptic meningitis during the treatment of psoriatic arthritis. Case Rep Dermatol 2017; 9: 26-29.

15. Mount HR, Boyle SD. Aseptic and bacterial meningitis: evaluation, treatment, and prevention. Am Fam Physician 2017; 96: 314-322.

16. Pashankar D, McArdle M, Robinson A. Cotrimoxazole induced aseptic meningitis. Arch Dis Child 1995; 73: 257-258.
17. Jolles S, Sewell WC, Leighton C. Drug-induced aseptic meningitis. Drug Saf 2000; 22: 215-226.

18. Jan JE, Hill RH, Low MD. Cerebral complications in juvenile rheumatoid arthritis. Can Med Assoc J 1972 107: 623-625.

19. Bathon JM, Moreland LW, DiBartolomeo AG Inflammatory central nervous system involvement in rheumatoid arthritis. Semin Arthritis Rheum 1989; 18: 258-266.

20. Huys AML, Guerne PA, Horvath J. Rheumatoid meningitis occurring during adalimumab and methotrexate treatment. Joint Bone Spine 2012; 79 : 90-92.

21. Yelehe-Okouma M, Czmil-Garon J, Pape E, Petitpain N, Gillet P. Drug-induced aseptic meningitis: a minireview. Fundam Clin Pharmacol 2018; 32: 252-260. 